\title{
Force Characteristics Analysis for Linear Machine with DC Field Excitations
}

\author{
Preshant A/L Krishna ${ }^{1}$, Aravind $\mathrm{CV}^{1,{ }^{*}}$, Aminath Saadha ${ }^{1}$, and $F$. Azhar ${ }^{2}$ \\ ${ }^{1}$ School of Engineering, Taylor's University, Malaysia \\ ${ }^{2}$ Faculty of Engineering, Universiti Teknikal Malaysia Melaka, Malaysia
}

\begin{abstract}
In urban regions and particularly in developing countries such as Malaysia with its ever-growing transport sector, there is the need for energy efficient systems. In urban railway systems there is a requirement of frequent braking and start/stop motion, and energy is lost during these processes. To improve the issues of the conventional braking systems, particularly in Japan, they have introduced linear induction motor techniques. The drawbacks of this method, however, is the use of permanent magnets, which not only increase the weight of the entire system but also increases magnetic cogging. Hence an alternative is required which uses the same principles as Magnetic-Levitation but using a magnet-less system. Therefore, the objective of this research is to propose an electromagnetic rail brake system and to analyze the effect of replacing permanent magnets with a magnet-less braking systems to produce a significant amount of brake thrust as compared with the permanent magnet system. The modeling and performance analysis of the model is done using Finite Element Analysis (FEA). The mechanical aspects of the model are designed on Solidworks and then imported to JMAG Software to proceed with the electro-magnetic analysis of the model. There are 3 models developed: Base Model (steel), Permanent Magnet (PM) Model and DC Coil Model. The performance of the proposed 2D models developed is evaluated in terms of average force produced and motor constant square density. By comparing the values for the 3 models for the same case of 9A current supplied for a $0.1 \mathrm{~mm} / \mathrm{s}$ moving velocity, the base model, permanent magnet model and DC coil model produced an average force of $7.78 \mathrm{~N}, 7.55 \mathrm{~N}$, and $8.34 \mathrm{~N}$ respectively, however, with increase in DC current supplied to the DC coil model, the average force produced is increased to $13.32 \mathrm{~N}$. Thus, the advantage of the DC coil (magnet-less) model, is, that the force produced can be controlled by varying the number of turns in the coil (N) or the current supply to the coil (I) given by the which is the simple principles of a solenoid: Force $(\mathrm{mmf})=\mathrm{NI}$.
\end{abstract}

${ }^{1}$ Corresponding author: aravindcv@ieee.org 


\section{Introduction}

Conventionally brake systems use friction or mechanical traction to slow down or stop a moving object, but friction produces heat and thus heat losses. Friction generates heat, thus losing energy, and the frequent use of these type of friction based braking leads to wear and tear of brake pads, wheels, and tracks leading to the requirement of frequent maintenance of the system[1]. Disc brakes require extra materials such as oil or compressed air to force the brake lining on the wheels, reduce the kinetic force of the system. Apart from the mechanical stress on the system, installing disc brakes increases the weight of the entire system, but the application is useful only during braking conditions [2]. Figure 1 shows the example of Wheel-Thread Brake System and Axle-Mounted Disc Brake System mechanisms which are of adhesion types:

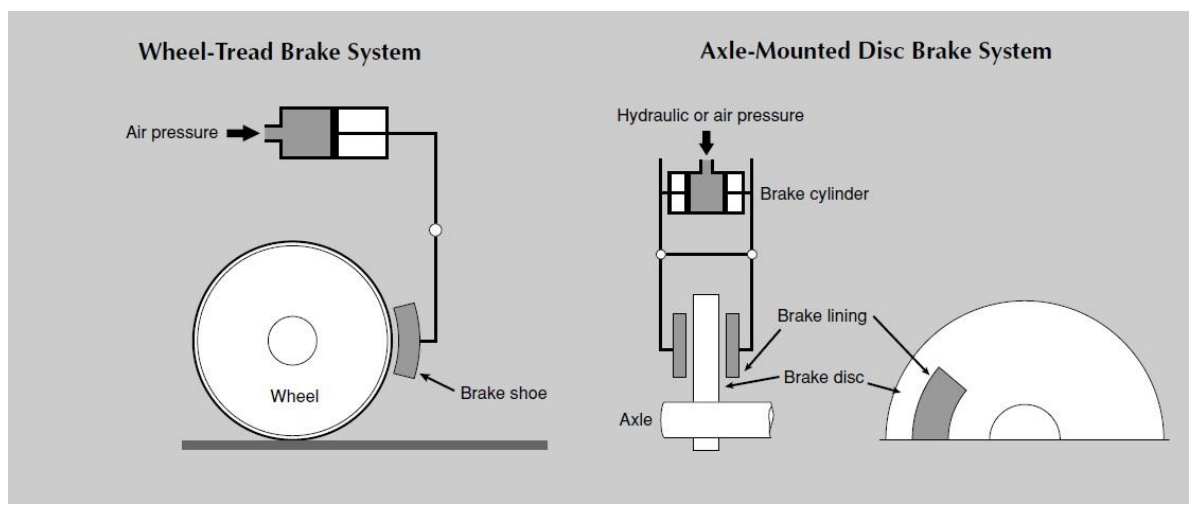

Fig. 1. Wheel-Thread Brake System and Axle-Mounted Disc Brake System [2]

To overcome this, electromagnetic brake systems that do not rely on wheel adhesion such as eddy current braking systems are introduced using electromagnetic induction principles, with the help of permanent magnets [3]. However, the drawback of this method is that, at lower speeds, the brake force is reduced without the ability to magnetically lock the load at a standstill, which poses a safety issue, and thus the conventional friction braking system has to be incorporated as well. Furthermore, the heat generated from the braking of these types of systems result in the overheating of the rail tracks causing the tracks to bend or deform. Another drawback with the use of permanent magnets is the increase in weight of the system as well as the cogging force induced. [1, 4]. Even hybrid linear motors have been researched and the effect of the core losses have been analyzed to be much more when there are permanent magnets introduced to the system[5]. Although research has been on the losses of permanent magnet systems, there is not a sufficient amount of literature and research data available for the application of magnet-less systems for railway braking aspects. [6]. Therefore, the objectives of this research are to propose an electromagnetic rail brake system through fixed DC excitation for rail brake systems which produce a significant amount of brake thrust and to analyze the effect of replacing permanent magnets with a magnet-less braking. Moreover, to compare the machine characteristics such as motor constant square density of the proposed model to the permanent magnet model, with respect to the conventional base model. 


\section{Research Methodology}

The research encompasses the design, optimization and prototype fabrication of linear electromagnetic braking using levitating principles for railway braking systems. Then the design is simulated and analyzed in software such as JMAG for electromechanical analysis and Finite Element Analysis and Solidworks for the mechanical modeling and analysis. The limitations and scope of the research are due to the restriction to the lab environment and the specifically fixed loading capacities of the prototype model. The flowchart of the flow of research methodology is given below in Figure 2.

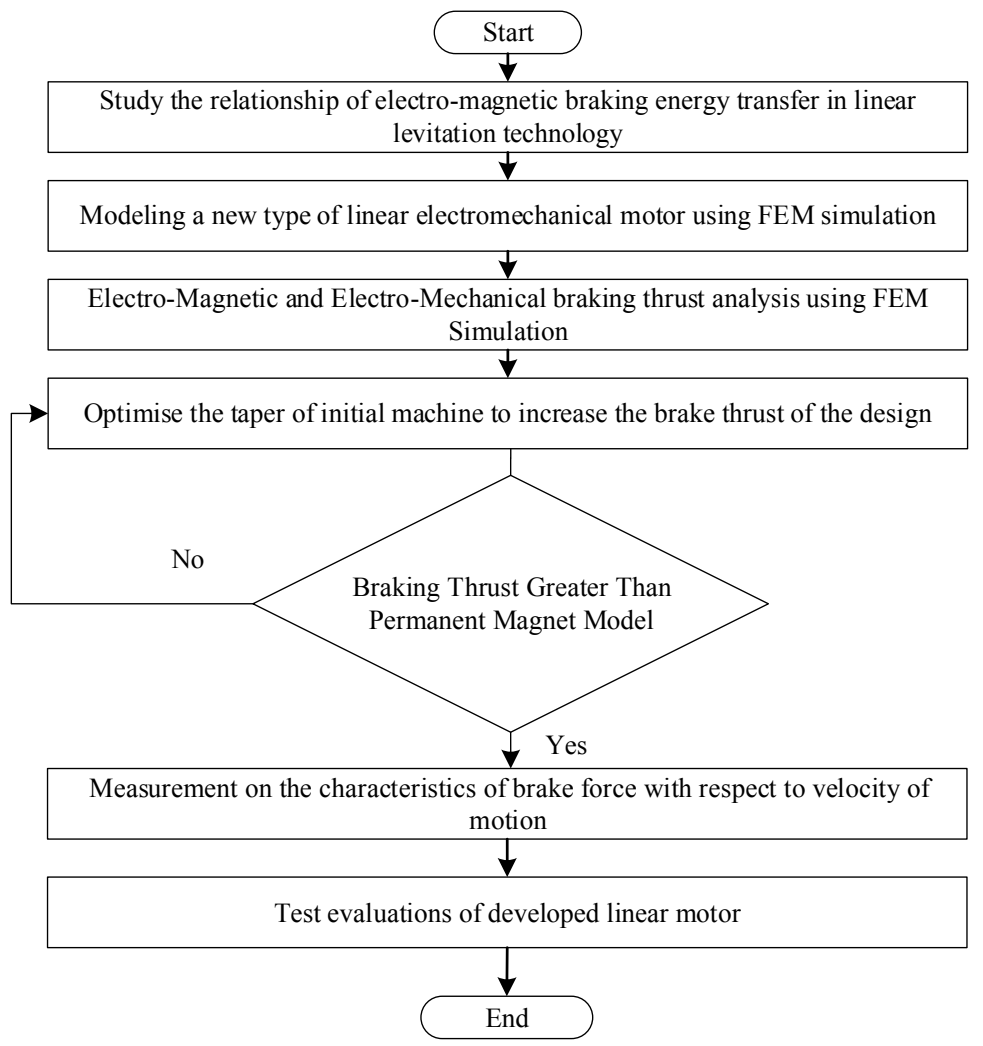

Fig. 2. Flowchart of Methodology

The relationship between the electrical and mechanical energy to the braking performance of the motor technology is mathematically developed. The purpose the study is to investigate the performance of the overall linear electromechanical technology for braking applications in railways. A set of coils energized attract steel and the flux flow in the air gap holds the rail and the track to be magnetically locked, which is the principle of the motor as seen in Figure 3. The flowchart for the overview of the design and development stages is shown below in Figure 4. 


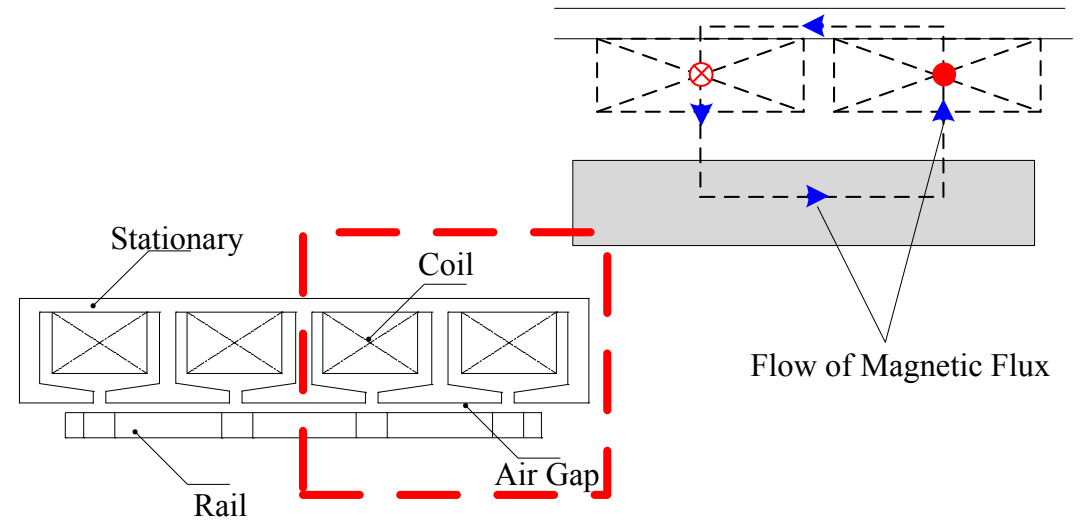

Fig. 3 Concept of Linear Switched Motor

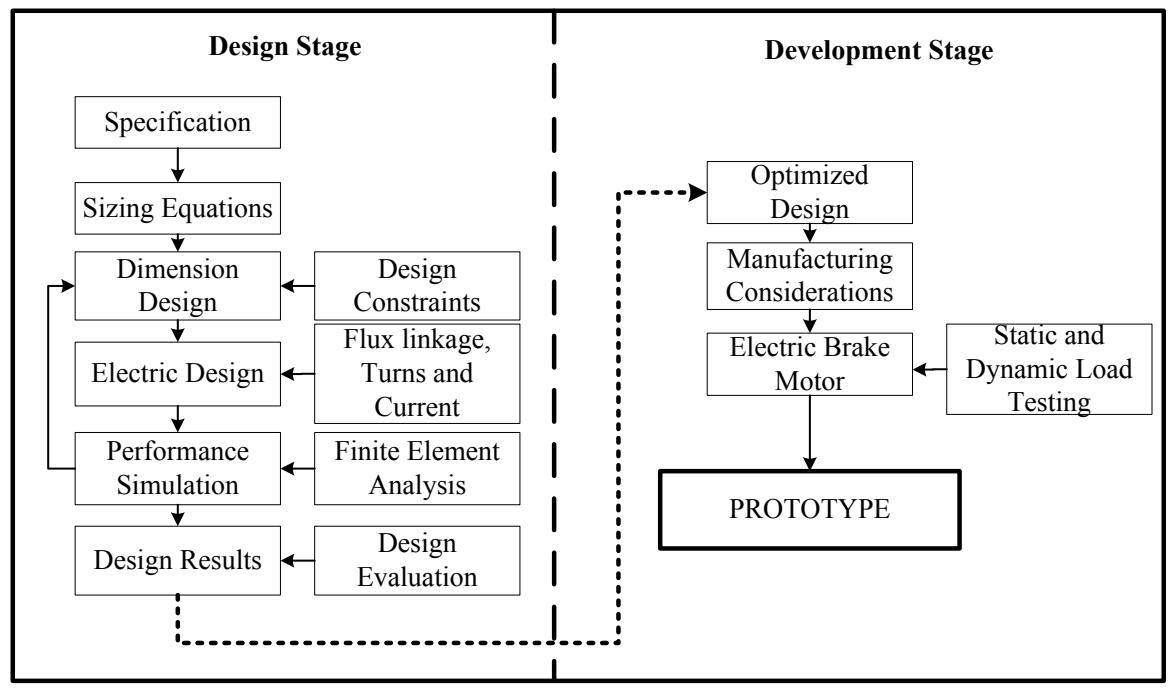

Fig. 4. Overview of Design and Development Stages

After the relationship is established, the next step is to model the new type of linear machine using numerical analysis tool. Design details are done analytically using calculations, before the modeling process. For the modeled prototype, the motor should produce maximum braking torque. The limits are set based on the sizing (height and width) calculations of the motor. Based on the analysis of the study and optimization done, the linear electromechanical motor prototype is fabricated to validate the proof of concept. Experimental analysis for the final prototype of the linear motor and the characteristic of the motor torque with a change in velocity is tested. Experimental analysis for the final prototype for the characteristics of the motor torque and speed is tested and compared to that of a linear permanent magnet motor. 


\subsection{Magnetic Circuit Analysis}

The magnetic flux density is given by the Eq. (1) [8,9].

$$
B=\mu_{o} H=\mu_{o}\left(\frac{N I}{x}\right)
$$

whereby, $B$ is flux density, $\mu o$ is Permeability of free space (air), $H$ is Magnetic flux intensity, $N$ is Number of turns of the coil and $I$ is the current carried by the coil.

The linkage flux is given by Eq. (2) [8,9].

$$
\psi=\phi N=\left(\frac{\mu_{o} A N^{2} I}{x}\right)
$$

The self-inductance $L$, is given by Eq. (3) [9].

$$
L=\left(\frac{\mu_{o} A N^{2}}{x}\right)
$$

Energy stored in a current carrying coil with the self-inductance of the coil $L$, and if there are no other current carrying coils in the system then magnetic flux $\phi$, is given by the Eq.(4) [8].

$$
\phi=I L
$$

Therefore the energy stored $W$, can be written as Eq. (5) below:[8]

$$
W=\frac{1}{2} L I^{2}
$$

If a system consists or more than one current-carrying coils that are magnetically linked to each other, the total force of the system is the sum of all the forces acting on the coils. In a multiple loop system, the force $F$, associated with linear motion is defined by a variable $x$, and is given as Eq. (6) $[8,9]$.

$$
F=\frac{\partial W}{\partial x}
$$

This can be substituted with Eqs.(1), (3), and(5) and rewritten to form the Eq.(7) [8,9].

$$
\begin{aligned}
& F=\frac{d}{d x}\left(\frac{1}{2} I^{2} L\right) \\
& F=\frac{d L}{d x}\left(\frac{1}{2} I^{2}\right) \\
& F=-\frac{1}{2} I^{2}\left(\frac{\mu_{o} A N^{2}}{x^{2}}\right) \\
& F=-\frac{1}{2 \mu_{o}}\left(\frac{\mu_{o} N I}{x}\right)^{2} A \\
& F=-\frac{B^{2} A}{2 \mu_{o}}
\end{aligned}
$$

whereby, $A$ is the cross section in the air gap, $B$ is the magnetic flux density and $\mu_{o}$ is the permeability. The negative sign indicates the fore deceases to $x$, thus it is an attractive force. This force is also knows as normal force which is the force of attraction between the 
armature core and the reaction rail of a linear motor through air gap. [8-10] Eq. (7) can also be written as Eq. (8) [8-10].

$$
F=\frac{B \cdot B \cdot A}{2 \mu_{o}}
$$

Since $\phi=B A$, for a region where the magnetic field is uniform and assuming the flux density has only one directional component $B$ with a uniform cross section $A$, by taking the perpendicular direction of $B$ we get the Eq.(9) below: [8,9]

$$
F=\frac{B \phi}{2 \mu_{o}}
$$

Thus the relationship can be established as Eq.(10) below:[8,9]

$$
\varphi=\frac{F_{m m f}}{\mathcal{R}}=\frac{N I}{\mathcal{R}}
$$

Thus is can be seen that keeping the area of cross section A constant, the force can be increased by increasing magnetic flux $\phi$. This can be achieved by increasing the number of turns of the coil $N$, increasing the current $I$, or by decreasing the reluctance $\mathcal{R}$. Reluctance is essentially, the amount resistance to the flow of the magnetic flux through the air gap. The effects of decreasing the reluctance can be more vividly visualized by rearranging the Eq. (9) to Eq. (10), to analyze the methods of manipulating the reluctance of the system [9].

$$
\mathcal{R}=\frac{m m f}{\varphi}=\frac{N I}{B A}
$$

\section{Design Aspects}

The components of the linear motor that is designed are the mover, stator, and armature coil. The armature coils are supplied by AC current supply and produce magnetic flux which produces a force when acting on the mover. The armature coil is made up of copper windings, while the mover and stator are steel. The mover and the stator are separated by an air gap. For the Permanent Magnet model, part of the steel is modeled to be cut out to fit permanent magnets on the stator. The same dimensions are used for the DC coil model, but instead of permanent magnets, copper coils are selected as the material. Figure 5(a) shows the front view of the base model and Figure 5(b) shows the PM/DC Coil model designed on Solidworks. The dimensions of the designed model for the permanent magnet/DC coil are given in Figure 6(a) and Figure 6(b) respectively for the front view and the auxiliary view. To compare the analysis between the comparison of the system with the permanent magnet and a magnet-less system using only coils, the dimensions for the permanent magnet slots are kept the same, and replaced by coils, for the DC coil model. The 3D models of the linear motor are designed on Solidworks and then imported to JMAG for the FEM analysis. Finite Element software is capable of accurately calculating magnetic fields and the related parameters for motor design. The analysis done is shown in Figure 7(a) and Figure 7(b) for the magnetic flux density and the magnetic field lines respectively: 


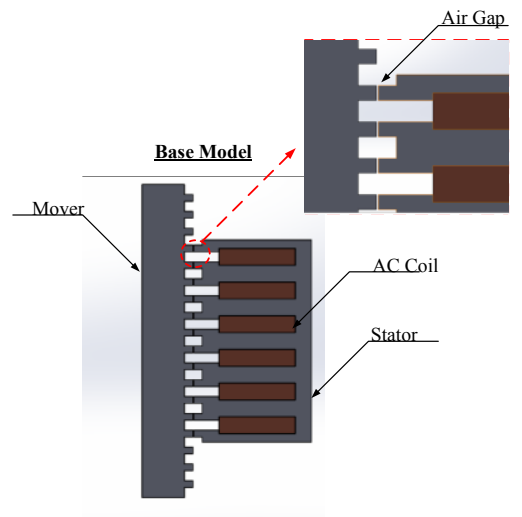

Fig. 5(a). Base Model Design

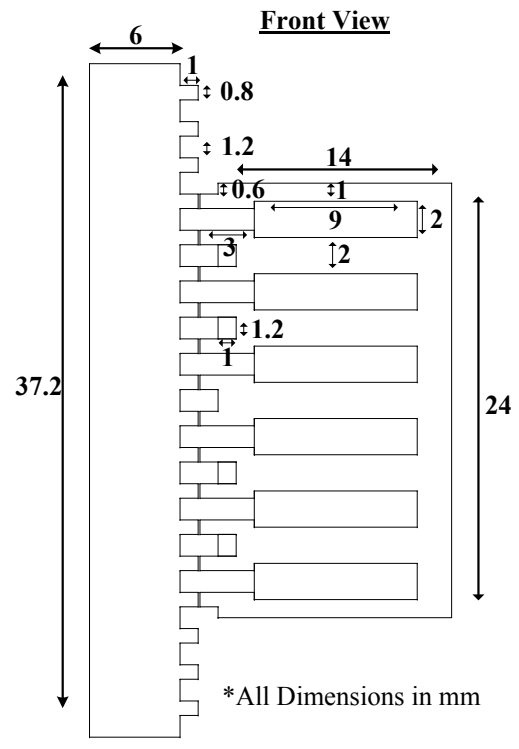

Fig. 6(a). Front View of Dimensions

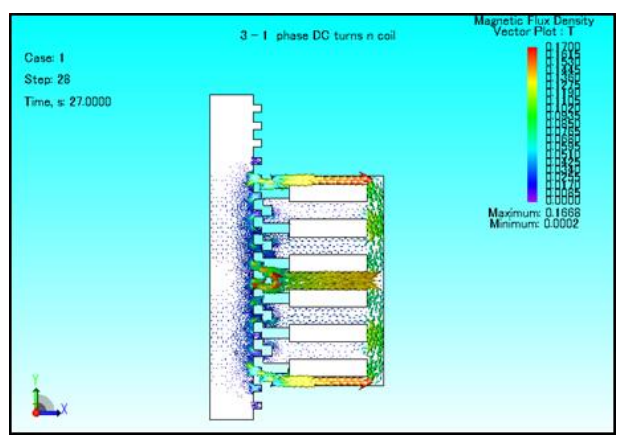

Fig. 7(a) Magnetic Flux Density

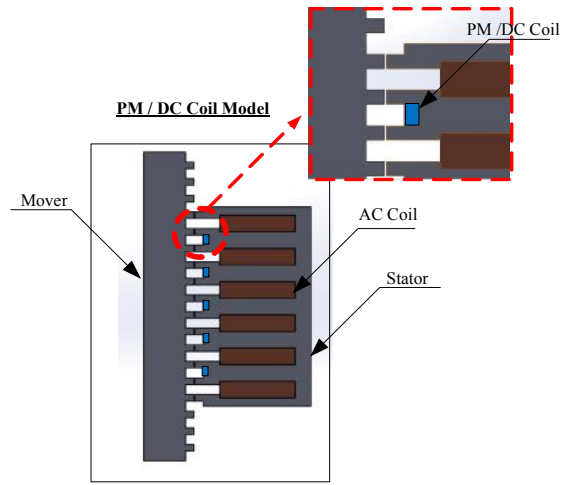

Fig. 5(b). PM / DC Coil Design

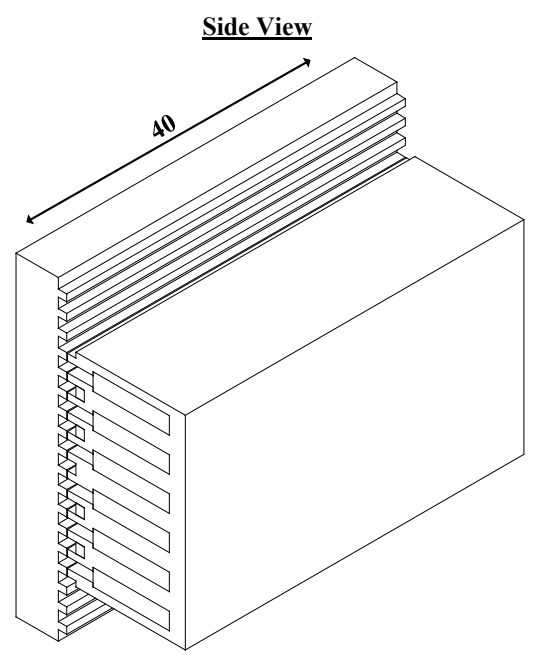

Fig. 6(b). Auxiliary View of Dimensions

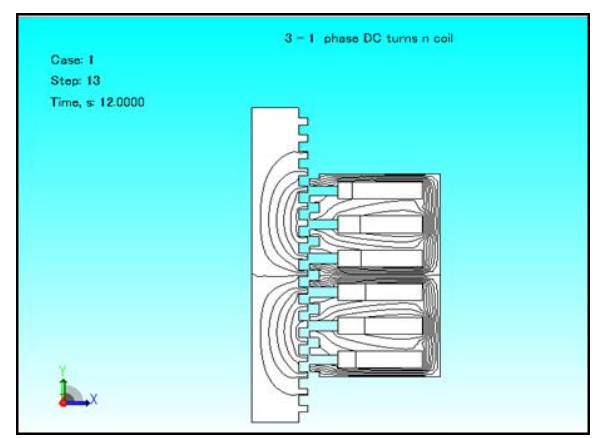

Fig. 7(b). Magnetic Flux Lines 
Thus, with these tools, the performance of the model is analyzed by analyzing the flow of the magnetic field lines and flux density. It is noted that the flux originates around the armature coil and flow through the mover, back into the stator, thus producing a force on the mover. With the step feature, the movement of the mover with each step with respect to time is be visualized and the change in magnetic flux is seen. The comparison FEM analysis is done for three models of linear motors: Base Model (steel), Permanent Magnet, Model and DC Coil Model (electromagnet). For the model with the DC coil, different values of current supply are also used at $1 \mathrm{~A}, 3 \mathrm{~A}$ and $5 \mathrm{~A}$ current for the DC coil, while the main AC supply to the stator is kept constant for all 3 models with 5A, 7A, and 9A current. For the 3 models, the average force values are tabulated for the different current values at various speeds with respect to time. The displacement values selected are $0.1 \mathrm{~mm} / \mathrm{s}$, $0.3 \mathrm{~mm} / \mathrm{s}, 0.5 \mathrm{~mm} / \mathrm{s}$. The force graph is obtained using JMAG and the values are tabulated and analyzed on the Origin software.

\subsection{Performance Index Calculation}

Thrust force produced by a machine is proportional to input power 'P', current ' $\mathrm{I}$ ' and volume ' $\mathrm{V}$ '. If the volume of a motor is larger, it has a larger area to accommodate a number of coil turns and/or coils of larger size, therefore more current and power can be injected into the machine to produce a higher thrust force. [11] Thus motor constant square density $G$, is used as one of the performance index in order to make valid comparisons between different models. This normalizes the factors such as volume, current and input power, to make a proper unbiased comparison between models. Thrust constant $k_{f}$, and motor constant $k_{m}$, are used as performance indices to normalize the current and input power of a motor respectively. The performance indexes are calculated using the equations Eq. (12) to Eq.(15) given below:[11]

$$
k_{f}=\frac{F}{I}
$$

Where $k_{f}$ is the thrust constant in (N/A), $F$ is the average thrust in $(\mathrm{N})$, and $I$ is the current injected to each coil (A). [11]

$$
P=I^{2} R
$$

Where $P$ is the input power in (W) and $R$ is the resistance of the coil.[11]

$$
k_{m}=\frac{F}{\sqrt{P}}
$$

Where $k_{m}$ is the motor constant in $(\mathrm{N} / \sqrt{W})$.[11]

$$
G=\frac{F^{2}}{P V}
$$

Where $G$ is the motor constant square density in $\left(\mathrm{N}^{2} /\left(\mathrm{Wm}^{3}\right)\right)$, and $V$ is the volume of the motor in $\left(\mathrm{m}^{3}\right)$.[11] 


\section{Results and Discussion}

To find the average force value for each case, from the data obtained in JMAG, the force graph for each case is integrated to find the area under the graph. The integrated values for each case in the 3 models are then tabulated and plotted on the same graph, thus the average force values for each model and each case is comparatively analyzed. Table 1 shows the data collected from the simulation done on the average force produced for each model for various cases:

Table 1. Average force produced for various cases of different models

\begin{tabular}{|c|c|c|c|c|c|c|c|c|c|c|}
\hline \multirow{2}{*}{\multicolumn{2}{|c|}{$\begin{array}{c}\text { AC } \\
\text { Supply } \\
\text { Velocity } \\
\text { (mm/s) } \\
\end{array}$}} & \multicolumn{3}{|c|}{$5 \mathrm{~A}$} & \multicolumn{3}{|c|}{$7 \mathrm{~A}$} & \multicolumn{3}{|c|}{ 9A } \\
\hline & & 0.1 & 0.3 & 0.5 & 0.1 & 0.3 & 0.5 & 0.1 & 0.3 & 0.5 \\
\hline \multirow{5}{*}{ Z } & Base & 5.87 & 5.87 & 5.36 & 7.10 & 7.10 & 6.46 & 7.78 & 7.75 & 7.08 \\
\hline & $\mathbf{P M}$ & 5.65 & 5.65 & 5.15 & 6.87 & 6.87 & 6.24 & 7.54 & 7.54 & 6.85 \\
\hline & $\begin{array}{c}\text { Coil } \\
1 A\end{array}$ & 6.57 & 6.57 & 6.00 & 7.71 & 7.72 & 7.02 & 8.34 & 8.34 & 7.57 \\
\hline & $\begin{array}{c}\text { Coil } \\
\text { 3A } \\
\end{array}$ & 8.85 & 8.85 & 8.08 & 9.82 & 9.82 & 8.96 & 10.3 & 10.38 & 9.48 \\
\hline & $\begin{array}{c}\text { Coil } \\
5 \mathrm{~A}\end{array}$ & 12.03 & 12.04 & 11.03 & 12.82 & 12.83 & 11.78 & 13.32 & 13.32 & 12.24 \\
\hline
\end{tabular}

Table 2 shows the graphical comparison of Velocity Vs Average Force produced by the 3 models for the supply AC current of 5A, 7A, and 9A:

Table 2. Comparison of Velocity Vs Force for Different Models

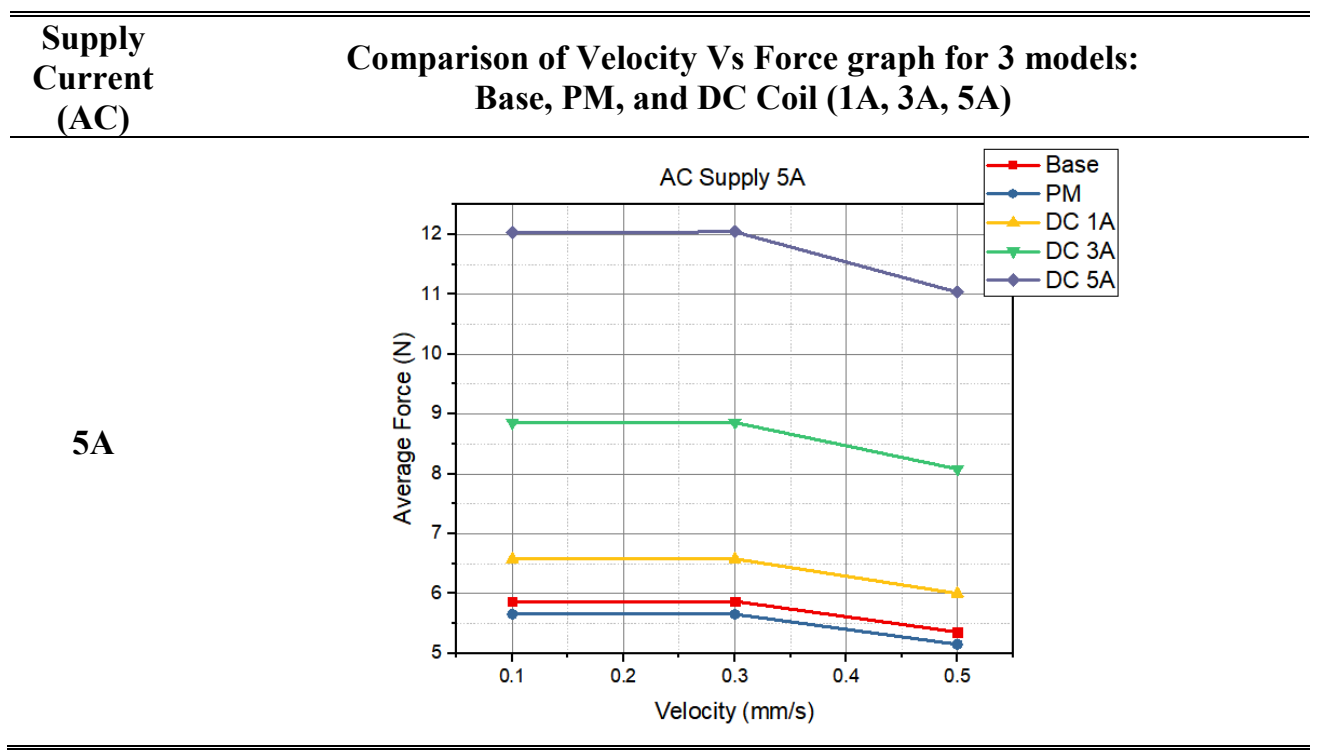


7A

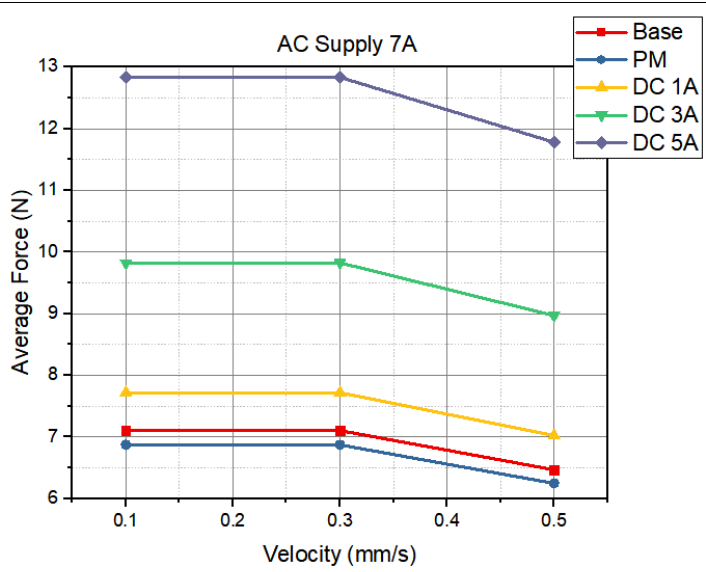

9A

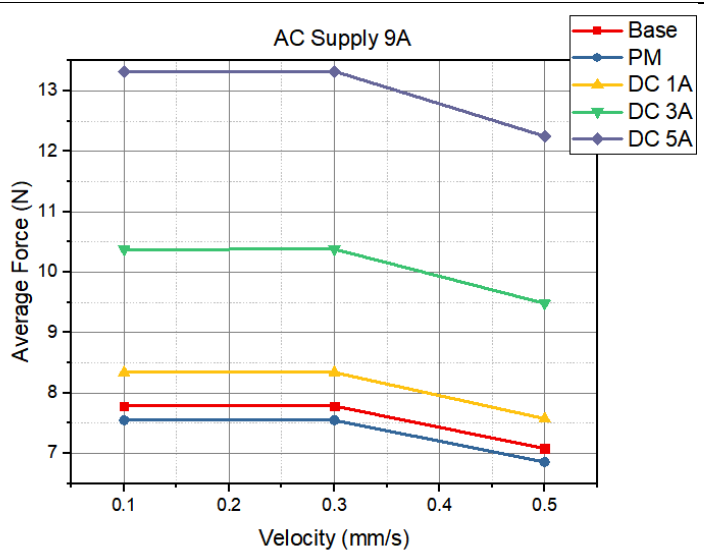

It is realized that, with varying the current in the DC coil, the brake force produced can be altered, without disrupting the main AC supply. Thus this gives a better range of force application that can be used for various brake force requirements. DC coils are used instead of permanent magnets for the proposed model, to curb the issues with magnetic cogging and to reduce the weight of the system. It also provides a wider range of operability compared to the permanent magnet as the magnetic field intensity of the coil can be increased or decreased depending on brake force requirement, but for permanent magnets, the force produced is constant and deteriorating over time. However, AC coil main supply to the system is still needed, to operate the railway system, as any unforeseen disruptions in the supply can be recovered by the AC supply, instead of affecting the entire system. The forces produced, however, are for the motoring condition, but if the polarity is reversed, the values obtained are for the braking condition. Since, the positive values indicate accelerating velocity and the negative values indicate decelerating velocity, hence braking condition. By combining the two conditions for the motoring and braking applications, the speed-force characteristics of the models is obtained. The speed-force characteristics of the different models are shown in Figure 8. The DC coil model produced an over higher average force when compared with the other models. As seen in the graph, by increasing the current value of the coil, there is an increase in the average force. Table 3 below shows the performance indexes for the research 


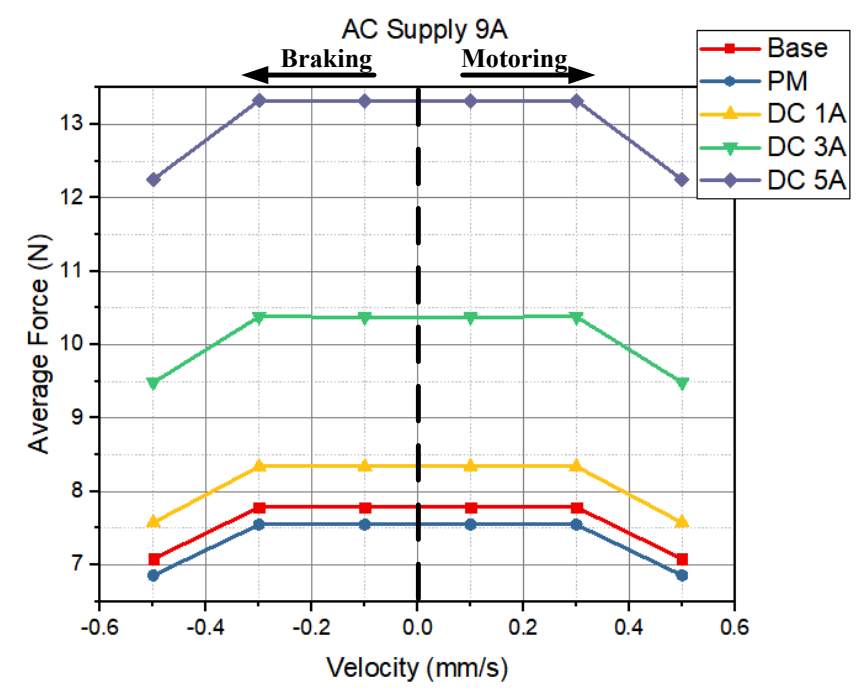

Fig. 8 Speed-Force Characteristic of the Different Models

Table 3. Parameters of Models

\begin{tabular}{|c|c|c|c|c|c|c|}
\hline \multirow{2}{*}{ Par. } & \multirow{2}{*}{ Unit } & \multirow{2}{*}{$\begin{array}{c}\text { Base } \\
\text { Model }\end{array}$} & \multirow{2}{*}{$\begin{array}{c}\text { PM } \\
\text { Model }\end{array}$} & \multicolumn{3}{|c|}{ DC Coil Model } \\
\hline $\mathrm{k}_{\mathrm{f}}$ & $\mathrm{N} / \mathrm{A}$ & 0.86 & 0.83 & 0.92 & 1.15 & 1.48 \\
\hline $\mathrm{k}_{\mathrm{m}}$ & $\mathrm{N} / \mathrm{A} / \sqrt{W}$ & 0.96 & 0.93 & 1.03 & 1.28 & 1.65 \\
\hline $\mathrm{G}$ & $(\mathrm{N} / \mathrm{A} / \sqrt{W})^{2} / \mathrm{mm} 3$ & $4.63 \mathrm{E}-05$ & $\begin{array}{c}4.36 \mathrm{E}- \\
05\end{array}$ & $5.32 \mathrm{E}-05$ & $8.23 \mathrm{E}-05$ & $1.36 \mathrm{E}-04$ \\
\hline
\end{tabular}

\section{Conclusion}

In conclusion, 3 models are developed: Base model, Permanent Magnet model, and the DC Coil model, whereby the average force produced and motor constant square density for each model for various moving velocities are compared and analyzed. The models produced an average force of $7.78 \mathrm{~N}, 7.55 \mathrm{~N}$, and $8.34 \mathrm{~N}$ for the base model, permanent magnet model, and DC coil model respectively. Comparing the characteristics of the models with respect to the base model, the permanent magnet model produced $5.98 \%$ decrease, while there is an increase of the DC coil model ranging from $14 \%$ to $192 \%$ with the increase of DC current supplied, for the motor constant square density.

\section{Acknowledgments}

The authors wish to express financial support of the project through the Taylor's Research Grant Scheme TRGS/MFS/1/2016/SOE/005. 


\section{References}

[1] R. C. Sharma, M. Dhingra, Braking Systems in Railway Vehicles, Int. J. Eng. Res. Technol., 4, no. 1, pp. 206-211, 2015

[2] I. Hasegawa and S. Uchida, Braking Systems, Japan Ralw. Transp. Rev. 20, vol. 7, no. June, pp. 52-59, (1999)

[3] C. Aravind, I. Khumira, R.N. Firdaus, Fairul.A, Single phase flux switching DC linear Actuator, Special issue pp.28-39 Journal of Science and Technology, 1, pp. 28-39, (2013)

[4] Y. Sakamoto, T. Kashiwagi, T. Sasakawa, N. Fujii, Linear eddy current brake for railway vehicles using dynamic braking, Proc. 2008 Int. Conf. Electr. Mach. ICEM'08, pp. 1-6, (2008).

[5] A. Aravind, V. R. Akilesh, S. Gunaseelan, S. Ganesh, Eddy Current Embedded Conventional, Natl. Conf. Trends Automotive. Parts Syst. Appl., 5, no. 7, pp. 7-12, (2016)

[6] M. Manna, S. Marwaha, M. Marwaha, Performance Optimization of Linear Induction Motor by Eddy Current and Flux Density Distribution Analysis, Journal of Engineering Science and Technology, 6, no. 6, pp. 769-776, 2011

[7] Shibly Ahmed Al-samarraie, Yasir Khuhair Abbas, Manifold based controller design for linear systems, Journal of Engineering Science and Technology, 8, no.6, 723-740 (2013)

[8] Q. A. Hasan, W. H. Wan Badaruzzaman, Ahmed W. Al-Zand, Azrul A. Mutalib, Finite element analysis of tapered composite plate girder with a non-linear varying web depth, Journal of Engineering Science and Technology, 12, no.11, 2839-2854 (2017)

[9] Y. Wang, J. Miao, Y. Wei, The research of traction motor energy-saving regenerative braking control technology, 2010 Int. Conf. Intell. Comput. Technol. Autom. ICICTA 2010, 3, pp. 930-933, (2010)

[10] I. Boldea, S. Nasar, Linear Electric Actuators and Generators. Cambridge, GBR: Cambridge University Press, (2009)

[11] H. Toliyat, G. Kliman, Handbook of Electrical Motors. Electrical and Computer Engineering. CRC Press, pp. 1-24, (2004)

[12] Rajesh V.R., Biju T.Kuzhiveli, Modelling and failure analysis of flexure springs for a stirling cryocooler, Journal of Engineering Science and Technology, 12, no.4, 888-897 (2017)

[13] F. Azhar bin Abdul Shukor, H. Wakiwaka, K. Tashiro, M. Nirei, Design and Performance Index comparison of the Permanent Magnet Linear Motor, Progress In Electromagnetics Research M, 43, pp. 101-108, (2015) 